\title{
Aktuelle Asthma-Übersicht
}

Vist orab: Diese 2. Auflage ist gegenüber der 1. Auflage von 1997 aktualisiert und das Werk gehört damit zu den besten aktuellen Informationsquellen über das Krankheitsbild Asthma.

Epidemiologie, Ursachen und $\mathrm{Pa}$ thophysiologie der Erkrankung sind ausführlich dargestellt. Neben einem Abriss der diagnostischen Maßnahmen ist der Schwerpunkt des Werkes die Asthmatherapie. Hierzu wird die Pharmakologie der einzelnen Wirkstoffe eingehend erläutert, inklusive der neueren Leukotrienantagonisten. Das Thema Churg-Strauss-Syndrom als medikamentöse Nebenwirkung nimmt immerhin 20 Seiten ein. Die Behandlung von Asthma im Erwachsenensowie im Kindes- und Jugendlichenalter wird getrennt und unter Berücksichtigung der aktuellen Therapieempfehlungen erläutert. Eigene Kapitel sind der Behandlung des Aspirin-induzierten Asthma bronchiale wie auch des Anstrengungsasthmas gewidmet. Die noch nicht verfügbaren Anti-IgE-Antikörper sind bereits mit einem Kapitel abgehandelt. Auf die Notwendigkeit einer gemeinsamen Behandlung von Rhinitis und Asthma wird hingewiesen. Schließlich werden die Inhalationstherapie und die Möglichkeiten einer ComplianceVerbesserung dargestellt.
Bei kritischer Durchsicht wird für den Leser naturgemäß die eine oder andere Formulierung nicht sofort zu akzeptieren sein. So verwundert das „nokturnale“ Asthma, gemeint ist das nächtliche Asthma. Der erhebliche Umfang der Leukotriene bzw. ihre Antagonisten betreffenden $\mathrm{Ab}$ schnitte scheint der Bedeutung der Substanzen nicht angemessen $\mathrm{zu}$ sein. Auch scheint die Bewertung der therapeutischen Maßnahmen nicht immer ganz ausgewogen. Unerfreulich ist die nicht sachgerechte Darstellung der spezifischen Immuntherapie. Kritisch anzumerken ist auch, dass auf dem Einband nur der Herausgeber genannt ist, nicht aber die stattliche Liste der Koautoren.

Insgesamt kann das Werk aber zur Information über Asthma bronchiale empfohlen werden, für Ärzte, Studierende und auch für interessierte Laien mit vertieftem Interesse an diesem häufigen Krankheitsbild.

Prof. Dr. Gerhard SchultzeWerninghaus, Bochum

Kroegel C, Hrsg. Asthma bronchiale. Pathogenetische Grundlagen, Diagnostik, Therapie. Stuttgart, New York: Georg Thieme Verlag, 2002. 24,95€.

ISBN 3-1310-4732-1

\section{Allergie ganz ungewöhnlich}

D as Buch „Allergie - ganz einfach“ erscheint in der 5. Auflage. Es hat nichts von seinem Charme verloren, sondern ist lediglich ergänzt und erweitert worden - beispielsweise durch das Kapitel zur Epidemiologie der Allergie mit den bekannten Unterschieden zwischen Stadt und Land, die Hygienehypothese sowie neue Allergene (z. B. Anisakis). Auch neue Zeichnungen sind ent- halten. „Allergie - ganz einfach“ ist das ungewöhnlichste Allergiebuch für Jung und Alt, Laien und Experten. Es gehört einfach dazu!

Prof. Dr. Dr. Johannes Ring,

München

Wahl R. Allergie - ganz einfach. Deisenhofen: Dustri-Verlag, 2001. 11,30 €.

ISBN 3-8718-5311-9 